\title{
Scanning laser densitometry in visual acuity loss of unknown origin
}

\author{
P J DeLint, J E E Keunen, A T A Liem, D van Norren
}

\begin{abstract}
Aim-To assess foveal cone photoreceptor function in patients with unexplained loss of central visual acuity.

Methods-Testing of foveal cone photoreceptor function was performed using scanning laser densitometry, colour matching (Rayleigh equation), and pattern electroretinography (ERG). Standard tests included full field ERG, electrooculography, visual evoked potentials, static perimetry, and fluorescein angiography.

Results-Decreased foveal cone photopigment density and abnormal pattern ERG were found in three patients. Results of colour matching were not unequivocal.

Conclusion-Testing of foveal cone photoreceptor function using scanning laser densitometry may determine the location of pathological changes in certain patients with unexplained visual loss.

(Br F Ophthalmol 1996;80:1051-1054)
\end{abstract}

Occasionally, patients may have a loss of central visual acuity without the cause being determined, even after an extensive examination. Examination generally includes best corrected central visual acuity, slit-lamp examination of the ocular media, direct and indirect ophthalmoscopy, visual field testing, fluorescein angiography, colour vision testing, electroretinography (ERG), electro-oculography (EOG), and visual evoked potentials (VEPs). Additional tests have been described in the literature. Matthews and colleagues ${ }^{1}$ used a focal ERG and extended colour vision tests. Two of five patients were found to have a red/green axis of confusion and all had reduced foveal cone ERG amplitudes, indicating that a foveal cone dysfunction was the cause of the loss of central visual acuity. Rowe et $a l^{2}$ reported reduced full field ERG amplitudes in six patients with unexplained acuity loss, and a colour vision disturbance in four of the six patients. All patients in these two studies had reduced photopic ERG amplitudes and most showed an impairment of colour vision. This suggests that there was a functional disturbance of the cone photoreceptors.

Our aim was to investigate a group of patients with a loss of central vision of unknown origin with a scanning laser densitometer. This is a sensitive technique to assess photopigment density in order to detect disturbances of foveal photoreceptor function..$^{3-8}$ In three patients with reduced photopigment density, we recorded the pattern ERG, ${ }^{910}$ and obtained colour matches.

\section{Materials and methods}

Fourteen patients between the ages of 16 and 52 years, with decreased central vision of unexplained aetiology, were examined over the past 7 years. The patients had noticed sudden or slow progressive loss of central vision. None of the patients had a history of drug ingestion, diabetes, neurological abnormalities, or a family history of ocular diseases. Patients were examined by at least two ophthalmologists before they entered the present study. Tests included best corrected Snellen visual acuity, visual field defects by the Peritest ${ }^{11}$ (Rodenstock), multiple stimulus static perimetry (stimulus size 30 minutes with 151 positions), colour vision defects by using the Ishihara, the Farnsworth Panel D-15 test, and colour matching. Ophthalmic examination also included ocular media opacities by slit-lamp examination and fundus abnormalities by direct and indirect ophthalmoscopy. By testing 52 healthy subjects Peritest normal values were established. A visual field defect was defined as more than $4 \mathrm{~dB}$ sensitivity loss above normal threshold and the normal foveal sensitivity was 1.0. None of the patients had nystagmus, strabismus, abnormal pupillary responses, or media opacities. Fundus examination revealed no abnormalities of the optic disc, vessels, or periphery. Fluorescein angiography, full field ERG, and EOG testing were performed in a number of patients, as was VEP with Ganzfeld and checkerboard pattern.

Retinal densitometry was performed with a scanning laser densitometer (SLD) ${ }^{12} 13$ covering a retinal area of $22^{\circ} \times 18^{\circ}$ with the fovea as centre. In retinal densitometry visual pigment density is calculated by taking the logarithm of the ratio of light reflection between a fully light adapted and fully dark adapted fundus. The implicit assumption is that the difference in the two images is only caused by the appearance of visual pigment. After each patient gave her or his written informed consent, the pupils were dilated with two drops of tropicamide $1 \%$. A bite board with a dental compound and two forehead rests ensured proper fixation of the patient's head. In necessary cases, refraction was corrected. One eye of each patient was examined by SLD. Images $(256 \times 256$ pixels $)$ obtained during light and dark adaptation were stored and later analysed. The SLD protocol lasted 12 minutes starting with a 2 minute bleach period, followed by image acquisition during 8 minutes of dark adaptation, and ending with a second 2 minute bleach.

The macular region $\left(8^{\circ} \times 6^{\circ}\right)$ was selected for further analysis. The centre of the fovea was located by using the perimacular reflection and 
the relatively dark appearance of the foveola, due to high absorbance at $514 \mathrm{~nm}$ of melanin and macular pigment. The mean foveal photopigment density was determined in the central $2^{\circ} \times 2^{\circ}$ area. The optical density in this area is almost entirely due to photopigment contained in the cones; these foveal cones primarily determine central visual acuity.

The mean foveal photopigment cone density for a healthy control group of 15 subjects (mean age 28 (1 SD 12) years) was 0.38 (2 SD $0.10)$.

Three patients with reduced foveal photopigment density were asked to have a pattern ERG test performed elsewhere; this technique is not available in our clinic. A checkerboard pattern (block size 60 minutes) with a frequency of $2 \mathrm{~Hz}$, and a mean luminance of 150 lux covering a visual angle of $18^{\circ} \times 16^{\circ}$, was presented to undilated eyes to elicit a response. ${ }^{14}$ Checkerboard contrast was $100 \%$. Outside the pattern field, a surround field was used to eliminate stray light responses. Luminance of the surrounding field was equal to or greater than the white checkers. Normal pattern ERG amplitude was between 2 and $5 \mu \mathrm{V}$, and normal implicit times of $P_{50}$ was between 55 and $65 \mathrm{~ms}$. A smaller stimulus than $18^{\circ} \times 16^{\circ}$ would be preferable to investigate the retina responsible for reduced visual acuity, but this would cause the signal to noise ratio to drop below the level where it can reliably be recorded. The pattern ERG was recorded in the same eye that was used for densitometry.

The Rayleigh equation was obtained by colour matching with a Nagel type I anomaloscope by testing, with reduced photopigment density, with a standard method. ${ }^{15}$ Midmatching points of the patients were assessed at a constant yellow scale setting of 12.5. On our anomaloscope, the normal range includes the red-green scale 37-45; the range for pseudoprotanomaly is between 46 and 60 .

\section{Results}

Fourteen patients were tested with retinal densitometry. Three patients had reduced foveal cone photopigment density. These three, who will be discussed in more detail, were further tested and were found to have abnormal pattern ERGs. Two of three patients had a colour match shifted to red. The remaining 11 patients had normal foveal photopigment densities.

\section{CASE REPORTS}

Patient 1

This patient was a 19-year-old man who had no ophthalmic complaints. His reduced visual acuity was noted during testing for his driver's licence. Visual acuity, with best refractive correction of the right and left eye, respectively, was 0.4 and 0.5 . There was no family history of retinal pathology. The media were clear and funduscopy revealed no abnormalities. Fluorescein angiography showed no pigment alterations, a dark choroid, or other abnormalities. The patient had no visual field defects. Eye movement and fixation were normal. Pattern and Ganzfeld VEPs had normal amplitudes and normal latency in both eyes. The patient made one mistake in the Ishihara test.

Scanning laser densitometry revealed reduced (0.05) foveal cone photopigment density (normal 0.28 to 0.45 ) in his left eye, as shown in Figure 1B. Pattern ERG showed normal amplitudes in the right eye of 2.21 to 4.27 $\mu \mathrm{V}$ and 2.39 to $3.10 \mu \mathrm{V}$ in the left eye. The implicit times $\left(P_{50}\right)$ were increased; the right eye was $67.4 \mathrm{~ms}$ and the left eye was $66.4 \mathrm{~ms}$. Colour matching showed a pseudoprotanomaly. Colour midmatch points for his right and left eye were 52 .

\section{Patient 2}

Patient 2 was a 46-year-old woman who had noted a progressive loss of visual acuity over the past 2.5 years. Visual acuity with best refractive correction was 0.16 for her right and 0.40 for her left eye. The media were clear. There was no family history of retinal pathology. Funduscopy revealed slight pigment reduction in the central fovea of both eyes. Static perimetry showed no abnormalities with a 30 minute object, but with a 10 minute object a relative central scotoma of $4 \mathrm{~dB}$ was found in both eyes. She showed a $4 \mathrm{~dB}$ loss in sensitivity in seven and eight out of 12 locations within the central $10^{\circ}$ in her right and left eye respectively and a reduced foveal sensitivity of 0.4 . A normal biphasic response was found in the dark adaptation. The fluorescein angiogram showed a mid-foveal window defect in the right eye and two parafoveal window defects in the left eye. Light to dark ratio in the EOG was normal for both eyes. VEP testing was not performed. Full field ERG showed normal photopic and scotopic amplitudes and implicit times.

Foveal photopigment density (Fig 1C) was subnormal $(0.20)$ in her left eye. Pattern ERG responses were absent in both eyes. Colour matching was normal, and measured a midmatch point of 42 and 43 for her right and left eye respectively.

\section{Patient 3}

This was a 29-year-old woman who had stable but reduced visual acuity for the past 7 years. In 1987 the patient had noticed a sudden loss of visual acuity in her left eye. She had no family history of retinal disease. Her visual acuity at that time was 0.5 in the right eye and 0.25 in the left eye. Visual field testing showed a relative central scotoma in her left eye, a $4 \mathrm{~dB}$ sensitivity loss in four out five locations within the central $5^{\circ}$ of the left eye using a 30 minute object and a reduced foveal sensitivity of 0.4 in her left eye. Colour vision testing using Ishihara plates and the Farnsworth-Munsell D-15 panel revealed no abnormalities. Pattern VEP testing resulted in normal $P_{100}$ latency times and amplitudes. Normal photopic and scotopic full field ERG responses were present in the left and right eye. Her left and right eye had central foveal pigment alterations, but no dark choroid or late phase glowing of retinal vessels on fluorescein angiography. Neurological examination found no evidence of multiple sclerosis. Seven years later we 

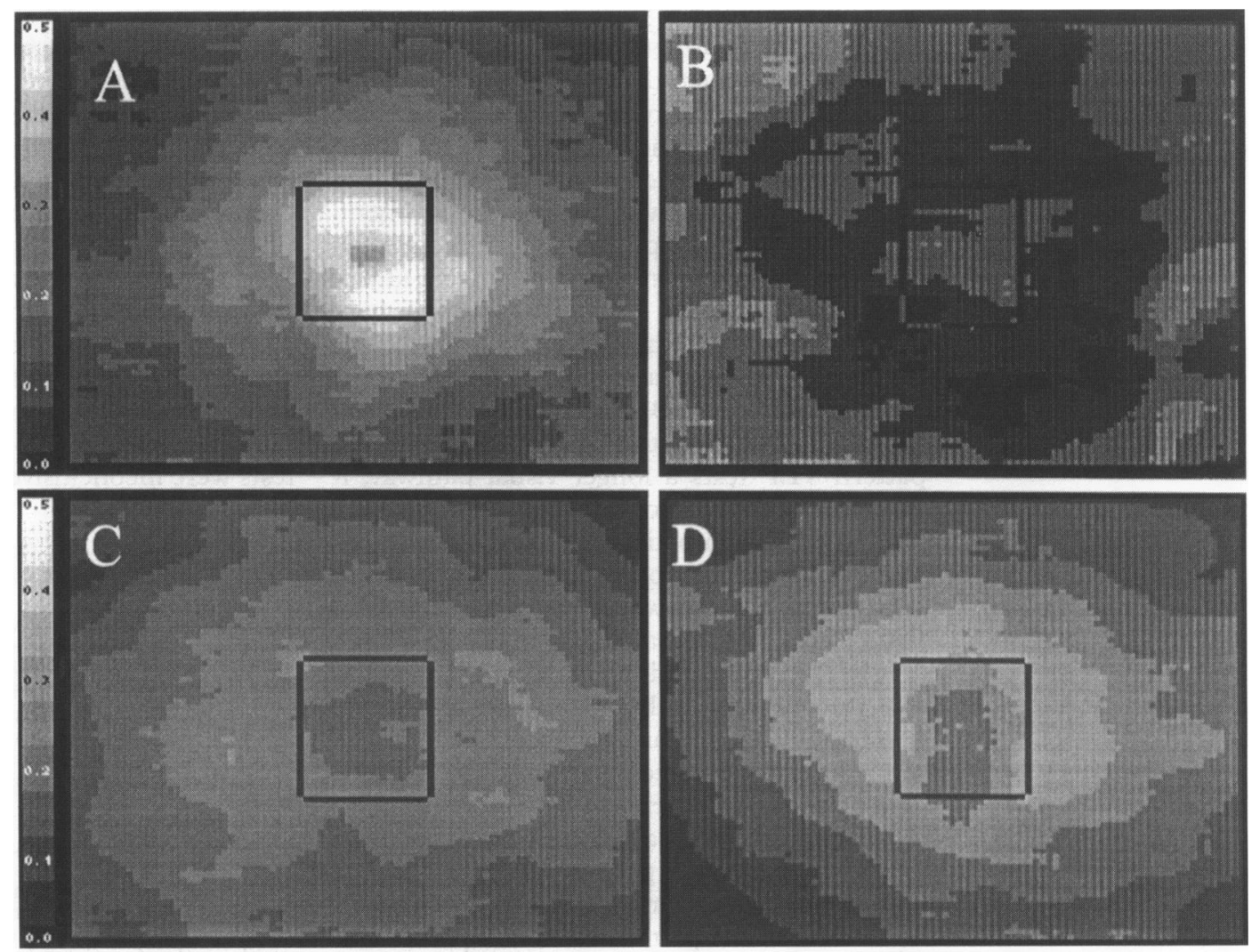

Figure 1 Visual pigment density images of the central macula, $8^{\circ} \times 6^{\circ}$. (A) Typical healthy subject; (B), (C), and (D) patients 1, 2, and 3 respectively. Shades of grey and corresponding visual pigment density values, ranging from 0.00 to 0.50 , are shown in the bars. Density values were smoothed and thresholded. Within the box, $a 2^{\circ} \times 2^{\circ}$ area, density values in $(B),(C)$, and $(D)$ are clearly lower than $(A)$.

found that her visual acuity was 0.5 in her right and left eyes. VEP latency and colour matching were unchanged. Full field ERG had normal photopic and scotopic responses and normal implicit times ( $28.5 \mathrm{~ms}$ in both eyes). Oscillatory potentials were found to be absent in her right and left eyes.

Scanning laser densitometry revealed reduced photopigment density (0.20) in her left eye for the central $2^{\circ}$, whereas density at $2^{\circ}$ eccentricity was found to be normal (0.33), as shown in Figure 1D. Pattern ERG responses were absent in both eyes. Colour matching showed a midmatch point (48) shifted to red for the right eye, and a widened range of 38 to 48 for the left eye.

\section{Discussion}

Scanning laser densitometry revealed decreased foveal cone photopigment density in three patients, indicating a cone photoreceptor dysfunction. This conclusion was corroborated by pattern ERG abnormalities in all three patients. Normal foveal density was found in 11 other patients with decreased central vision, making abnormalities at the photoreceptor level unlikely. Earlier extensive examinations had not explained the loss of central vision in any of the patients.

Causes for a reduced foveal cone photopigment density might be: (1) loss of cone photoreceptors and/or shortening of cone outer segments, (2) photoreceptor disorientation, (3) metabolic dysfunction of the cone photoreceptor-retinal pigment epithelium complex, or (4) high stray light levels. ${ }^{8}$
High stray light levels (cause 4) were unlikely, because the ocular media were clear and there were no changes on funduscopy which might have increased the stray light level. Furthermore, the retinal aperture of our confocal SLD is very small (0.43 degrees), reducing stray light considerably. ${ }^{16}$ Cause 3 was also unlikely, because a metabolic dysfunction of the cone photoreceptor-retinal pigment epithelium complex may result in retinal pigment epitheliopathy, which was not noted on funduscopy, except in patient 2. Yet, the EOG, reflecting the function of the retinal pigment epithelium, was normal in patient 2 .

Funduscopy and fluorescein angiography were normal, excluding a gross disorientation (for example, retinal folds) of the foveal cones. The pseudoprotanomaly (a colour match slightly shifted to red) noted in patients 1 and 3 , can be ascribed to a decreased effective optical cone photopigment density and may be caused by shortening or disorientation of photoreceptors. ${ }^{17}{ }^{18}$ Decreased foveal cone photopigment density may also be due to a reduced number of cones. This cause might apply to patient 2 who showed no pseudoprotanomaly.

A number of factors may cause an abnormal pattern ERG. We excluded a defocused retinal image caused by incorrect refraction. ${ }^{19} \mathrm{Un}$ steady eye fixation could also be excluded. Media opacities, which may cause contrast reduction, were not found. ${ }^{20}$

Most authors agree that the pattern ERG originates in the inner retinal layers, probably in the ganglion cells. However, a reduction in photoreceptor response or a decreased transfer 
of photoreceptor response to the neurons may also result in an abnormal pattern ERG.

Despite the fact that in all three patients deviations in pattern ERG were found, the interpretation is by no means clear. Increased implicit times recorded in patient 1 have been noted in patients with retinal detachment, inflammatory retinopathy, and refractive error, ${ }^{20}$ but there were no signs indicating any of these. Nevertheless, reduced photopigment density indicated a photoreceptor abnormality. Pattern VEP in patient 1 did not show prolonged latency. Because latency was only slightly increased in the pattern ERG and the pattern VEP tests a longer visual pathway, it may still be within normal limits. The normal photopic ERG in patient 2 at first sight seems to be in contradiction to her absent pattern ERG response. However, because full field ERG elicits an integrated response over a large area of the retina, and the cones in the macular area account for only $7 \%$ of the total cone population, ${ }^{21}$ macular abnormalities may be too subtle to be detected by a full field ERG. The pattern ERG seems to be a more sensitive test to detect macular disease than full field ERG. ${ }^{9}$ In patient 3 pattern VEP was absent with a normal pattern VEP. Lower signal to noise ratio in the pattern ERG compared with pattern VEP may be responsible for an absent or unrecordable pattern ERG response with normal VEP response. No pattern ERG or oscillatory potentials, which probably originate in the amacrine and interplexiform cells, ${ }^{20}$ were found in patient 3. Both indicate an inner retinal layer abnormality.

Prolonged latency in patient 1 and unrecordable pattern ERGs in patients 2 and 3 suggest different underlying pathologies. Although photopigment density images, shown in Figure 1 , also suggest that patient 1 may be different from patients 2 and 3, we need to test a larger number of these types of patients in the future to be more certain.

In the 11 patients with normal foveal photopigment densities it is seems less likely that there were foveal cone photoreceptor abnormalities in these patients causing loss of visual acuity. When the visual acuity of five patients with normal foveal photopigment density was retested at various distances, three patients appeared to have spontaneously recovered, strongly indicating a non-organic loss of visual acuity.

A more definite statement about photoreceptor function would have been possible if the focal ERG testing was performed. This technique was not available to us.

The differential diagnosis of the three patients with foveal cone dysfunctions might include various macular diseases such as emerging cone degenerations, like Stargardt's disease or progressive cone dystrophy, and emerging rod-cone degenerations. Stationary congenital cone dysfunction syndromes (such as incomplete achromatopsia) are unlikely as the visual acuity decreased at older age. There were also no signs of inflammation or of previous central serous chorioretinopathy. Yearly retinal function tests may finally lead to a definite diagnosis. We conclude that scanning laser densitometry may be a useful aid in the search for foveal cone photoreceptor abnormalities in patients with loss of visual acuity of unknown origin. SLD and pattern ERG distinguished between abnormal and normal foveal cone photoreceptor function, whereas other retinal tests were inconclusive in our patient group.

Supported by the F P Fischer Foundation.

1 Matthews PG, Sandberg MA, Berson EL. Foveal cone electroretinograms in patients with central visual loss of unexplained etiology. Arch Ophthalmol 1992;110:1568-70.

2 Rowe SE, Trobe JD, Sieving PA. Idiopathic photoreceptor Rowe SE, Trobe JD, Sieving PA. Idiopathic photoreceptor
dysfunction causes unexplained visual acuity loss in later dysfunction causes unexplained visual acuit
adulthood. Ophthalmology 1990;7:1632-7.

3 Carr RE, Ripps H. Siegel M, Weale RA. Visual functions in congenital night blindness. Invest Ophthalmol Vis Sci 1966; 5:508.

4 Van Meel GJ, van Norren D. Foveal densitometry as a diagnostic technique in Stargardt's disease. Am $\mathcal{f}$ Ophthalmol 1986;102:353.

5 Keunen JEE, van Everdingen JAM, Went LN, Oosterhuis $J A$, van Norren D. Color matching and foveal densitometry in patients and carriers of an $\mathrm{X}$-linked progressive cone in patients and carriers of an X-linked proge
dystrophy. Arch Ophthalmol 1990;108:1713.

6 Van Meel GJ, Keunen JEE, van Norren D, van de Kraats J. Scanning laser densitometry in the multiple evanescent Scanning laser densitometry in the muti
white dot syndrome. Retina 1993;13:29.

7 Ter Bruggen JP, van Meel GJ, Paridaens ADA, Tijssen CC, van Norren D. Foveal photopigment kineticsabnormality: an early sign in myotonic dystrophy? $\mathrm{Br} \mathcal{F}$ Ophthalmol 1992;76:594-7.

8 Liem ATA, Keunen JEE, van Norren D. Foveal densitometry in adult-onset diffuse drusen. Am $\mathcal{F}$ Ophthalmol 1992;114:149-57.

9 Arden GB, Carter RM, MacFarlan A. Pattern and Ganzfeld electroretinograms in macular disease. $\mathrm{Br} f$ Ophthalmol 1984;68:878-84.

10 Holder GE. Significance of abnormal pattern electroretinography in anterior visual pathway dysfunction. $\mathrm{Br} \mathcal{F}$ Ophthalmol 1987;71:166-71

11 Greve EL, Dannheim F, Bakker D. The Peritest, a new automatic and semi-automatic perimeter. Int Ophthalmol 1982;5:201-14.

12 Van Norren D, van de Kraats J. Imaging retinal densitometry with a confocal scanning laser ophthalmoscope. Vis Res 1989;29:1825-30.

13 van Meel GJ, van Norren D, van de Kraats J. Scanning laser densitometry in two patients with retinitis pigmentosa. Clin Vis Sci 1992;7:501.

14 Riemslag FCC. Retinal and occipital pattern evoked potentials. University of Amsterdam. Doctoral dissertation, 1986.

15 Pokorny J, Smith VC, Verriest G, Pickers AJLG. Congenital and acquired color vision defects. New York: Grune and Stratton, 1979:119-20.

16 Elsner AK, Burns SA, Hughes GW, Webb RH. Reflectometry with a scanning laser ophthalmoscope. Appl Optics 1992;31:3697-710.

17 Pokorny J, Smith VC, Ernest JT. Macular color vision defects. Specialized psychophysical testing in acquired and hereditary chorioretinal diseases. Int Ophthalmol Clin 1980; 20:53.

18 Keunen JEE, van Norren D, van Meel GJ. Density of foveal cone pigments at older age. Invest Ophthalmol Vis Sci 1987; 28:985.

19 Leipert KP, Gottlob I. Pattern electroretinogram: effects of miosis, accommodation, and focus. Doc Ophthalmol 1988; 67:335-46.

20 Berninger T, Arden GB. The pattern electroretinogram. In: Heckenlively JR, Arden GB, eds. Principles and practice of clinical electrophysiology of vision. St Louis: Mosby Year

1 Book, 1991 .

the layer of rods and cones in the human retina. Acta Ophthalmol 1935;6 (suppl):1. 